\title{
EL MARKETING PLAN
}

\author{
Rafael Antonio Reyes M.*
}

\section{Introducción}

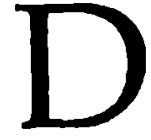

ado que lo único constante en el entorno empresarial es la dinámica de el cambio y que cada vez las estrategias cor porativas están siendo orientadas por las fuerzas del mercado se hace imperativo para las empresas un análisis del mismo y un ajuste de las estrategias bajo la perspectiva de la planificación mercadológica. Las aproximaciones intuitivas y empiricas sobre las ventas y administración de mercadeo son cada vez menos efectivas en la realidad actual.

Importancia de la planeación en márketing

Wada es más claro para los gerentes de ventas y mercadeo, el 1 nuevo desafío al que se enfrentan en la década de los noventa, década que se caracteriza por una nueva divisoria tanto a nivel nacional como internacional, estos cambios exigen una nueva orientación de los negocios, basada en la planificación corporativa y de mercadotecnia asi como de un nuevo rol de los gerentes de mercadeo y ventas. Este nuevo rol demanda para los gerentes en esta área mayor participación en los planes y actividades de campo, dejando atrás la usual postura contemplativa.

Hay ciertas fuerzas que están remodelando el papel de la mercadotecnia haciendo que ésta se oriente al concepto de planificación como base de toda gestión entre las cuales tenemos:

- Catedrático del Departamento de Administración de Empresas. 
a) Las nuevas tendencias en gustos, necesidades, medios ambientes y hábitos de vida

- Incorporación de la mujer al ámbito laboral.

- Hogares con dos fuentes de ingresos

- Reducción de la estructura familiar.

- Incremento de los matrimonios jóvenes

- Preocupación por la salud y lo natural

b) Desmasificación del mercado

Nos encontramos en una década de diversidad sin preceden tes, caracterizada por una serie de minimercados que no dejan de multiplicarse, basta con visualizar algunas categorias de productos como el de las bebidas gaseosas, cigarrillos, grasas comestibles, productos de café; sectores en los cuales encontramos distintos tipos de satisfactores para distintos tipos de consumidores.

\section{c) Cambios en lealtad de marcas}

La misma proliferación de productos y la aguda crisis socieconómica hace que el consumidor sea mucho más racional en su compra y busque mejores soluciones alternativas para satisfacer sus necesidades, buscando eficiencia y eficacia.

\section{d) Nuevas formas de comprar y pagar}

El proceso típico de compra-venta se vuelve cada día más personalizado y a la vez más complejo a través de el desarrollo e implementación de nuevas técnicas como es el caso de el Telemercado (uso planificado y sistemático de mercadeo); el mercado directo (Sistema interactivo que utiliza uno o más medios publicitarios para producir una respuesta medible y/o una transacción en cualquier sitio); mercadeo de base de datos (uso de la computadora para desarrollar perfiles detallados de personas y clientes, empleando características geográficas, y sicográficas), además tenemos que agregar el fenómeno de plastificación de dinero (tarjetas de crédito), que también se constituye como una nueva forma de comprar y pagar. 


\section{e) Competencia de precio a competencia sin precio}

Una de las estrategias competitivas más usuales, así como el argumento de venta más empleado por la fuerza de ventas ha sido competir vía precio. En la actualidad y en el período próximo futuro se están empleando nuevas armas competitivas que trascienden al precio como única forma de competir y de diferenciarse, así tenemos una gama de estrategias basadas en el merchandising, serviclo al cliente, posicionamiento, maximarketing igualmente todas las estrategias que pueden existir, basadas en cada una de las variables del márketing mix.

\section{f) Diversificación de canales de distribución}

Los cambios constantes en los consumidores y en los estilos de vida, exigen nuevas formas de pensar acerca de la distribución, lo cual implica plantearse estrategias no tradicionales de distribución que posibiliten una mejor cobertura de mercado; asi por ejemplo podemos encontrar en una farmacia desde cigarrillo hasta bebidas gaseosas y juguetes.

Las fuerzas antes mencionadas no son la totalidad, pero si son indicativas que el mercado está sufriendo cambios de vital importancia que significan un nuevo reto para la gestión de márketing. $Y$ es en este sentido que se antepone la importancia de la planificación de mercadeo para poder maximizar las oportunidades y minimizar las amenazas.

La Planeación como proceso trae los siguientes beneficios:

- Ayuda a los ejecutivos a pensar en el futuro.

- Es una herramienta coordinadora en todas las áreas de la empresa.

- Proporciona a los ejecutivos mejor sentido de responsabilidad.

- Prepara a la empresa para hacer frente a problemas en el futuro (gustos, hábitos, etc.)

- Proporciona estándares para control en la empresa.

- Toda la empresa afina detalladamente los Programas, Políticas, objetivos, etc. 
Para planear mercadológicamente debemos considerar los siguientes pasos básicos:

- Determinación del mercado

- Fijación de objetivos

- Determinar el Personal Administrativo con el cual se cuenta

- Preparar estrategias y programas para lograr los objetivos

- Preparación del plan definitivo

- Ejecución del plan

- Control del plan.

El planeamiento en mercado implica dos clases de decisiones de alta gerencia (la cual tiene que estar plenamente identificada): 1) determinar en que negocio se encontrará la organización en el futuro, mediante la definición de una estrategia corporativa que especifique los productos que serán generados y los mercados que abastecerá la organización; 2) la alta gerencia debe determinar el tipo de contribución que se espera rinda cada producto en la estrategia corporativa a largo plazo.

Queda de manifiesto que la planificación en mercadeo es importante porque nos permite visualizar el futuro y el lugar donde queremos estar, asi como lo que debemos hacer. Puesto que los ingresos de la empresa provienen de las ventas que no son el objetivo del mercadeo, sino la última consecuencia de un buen mercadeo, es necesario que los garanticemos mediante un adecuado proceso mercadotécnico.

Pasos para elaborar el márketing plan .

a) Coordinación entre los planes de mercadotecnia y los planes del negocio

El proceso de planificación en los negocios es un proceso continuo y comienza con la evaluación de la situación que la empresa enfrenta. Esto conduce a un examen de la misión y los objetivos de la empresa que pueden con el tiempo responder o no responder a los cambios. Según se determine a través de situaciones específicas. Se requieren las estrategias para lograr misiones y objetivos. Dichas 
estrategias se elaboran para las áreas de producto y de mercado que determinan la composición del negocio. La participación de la mercadotecnia en la planificación estratégica de los negocios es clave, asi tenemos:

1) Determinación de la misión de la organización.

El gerente de mercadeo es participe importante con quien se elabora los planes estratégicos.

2) Análisis de situación.

Es un contribuyente importante y uno de los principales beneficiarios de los resultados.

3) Objetivos y metas.

Participante clave con otros gerentes funcionales, incluyendo responsabilidad por la mediación de diversos indicadores del desempeño.

4) Estrategias.

Responsable de la estrategia de mercadeo y de la coordinación de los planes con otras estrategias funcionales.

5) desarrollo de Producto-Mercados.

Papel de liderazgo.

6) Distribución.

Responsabilidad primordial.

7) Calidad

Principal responsabilidad por la calidad.

8) Recursos humanos

Responsabilidad por el área funcional

Como se puede apreciar a responsabilidad de la gerencia de mercado en la planificación de los negocios es determinante para un plan corporativo congruente y funcional. Nunca se tiene que pensar ni mucho menos diseñar un plan mercadotécnico sin que antes exista una coordinación con el plan corporativo; esto es un defecto del que adolecen muchas empresas cuando tratan de iniciar un proceso de planificación. La sinergia en los planes es la 
piedra angular de éxito de una planeación corporativa y funcional efectiva.

Guia básica para coordinar los planes de negocios y de mercadotecnia

- ¿cuál es la misión y los objetivos de nuestra organización?

- ¿Cómo se divide nuestra organización en áreas de negocio?

- ¿Cuál es el plan de la alta dirección para nuestra unidad de negocios?

- ¿Cuáles son nuestros objetivos y estrategias para llevar a cabo los planes que la alta dirección tiene para nuestra unidad de negocios?

- ¿Las estrategias están logrando los resultados que deseamos lograr?

- ¿Nuestra estrategia de mercadeo es consistente con la estrategia de la unidad de negocios?

b) Análisis de situación (Datos básicos)

El establecer la coordinación entre los planes corporativos y los planes de mercadeo nos permite dar respuesta a dos interrogantes básicas que están intimamente relacionadas con las misiones de la empresa y que constituyen el punto de inicio de todo plan de márketing:

¿En qué negocios estamos?

¿Hacia qué mercados debemos dirigimos?

La definición de misiones y metas nos brindan un marco general dentro del cual se habrá de trabajar. El próximo paso consistirá en recopilar, analizar y evaluar los datos básicos.

La primera parte de los datos básicos consiste en una evaluación general a efecto de buscarle respuestas a la siguiente interrogante:

¿Dónde estamos ahora?

La respuesta a esta pregunta nos proveerá de datos primarios para la planificación posterior. 
La primera parte del análisis de situación se inicia tratando de dar respuesta a las siguientes preguntas:

- ¿Quiénes son nuestros consumidores actuales y potenciales? ¿Dónde están? ¿Cuáles son sus problemas, necesidades y deseos?

- ¿Qué beneficios puede proveer nuestra empresa, a través de sus productos/servicios a estos consumidores que ellos no pueden lograr a través de otra via?

- ¿Dónde estamos ahora y dónde deseamos estar dentro de " $X$ " años a partir de ahora?

- ¿Por qué deseamos estar ahí?

- ¿Cuáles son los principales obstáculos que hemos de preveer para llegar allí?

- Las estrategias que estamos implementando ahora, ¿nos permitirán llegar a donde deseamos?

- ¿Cuáles son nuestros principales competidores?

¿Cuál es su ventaja diferencial? ¿Cuál es nuestra opinión sobre sus estrategias y metas? ¿Cómo comparamos nuestros productos/servicios con los de ellos (producto, precio, plaza, promoción, servicio, etc.)?

- ¿cuáles son nuestras fortalezas y debilidades? ¿Cuáles son las de la competencia?

El siguiente paso del análisis de situación o monitoreo del entorno es segmentar los datos en áreas de interés que dependerán de las caracteristicas especificas de la empresa asi como las de los productos o servicios entregados. Sin embargo se pueden distinguir las siguientes áreas comunes a la mayoria de las empresas:

\section{a) Ventas}

- Análisis histórico de las ventas, en dinero, en unidades y por línea de productos para los últimos cinco años, incluyendo las variaciones por periodo (periodos pico y periodos valle).

- Actualizar y estandarizar los datos en períodos de análisis (semanal, mensual, trimestral, etc.). 
- Preparar pronósticos de venta, estimado como se prevee que terminará el período en valor y unidades.

- Establecer comparaciones entre lo pronosticado y realizado.

- Para medir las tendencias: diseñar un cuadro comparativo de ventas (unidades y dinero) desglosado.

- Productos

- Línea de productos

- Mercados

- territorios de venta

- Canales de distribución

- Clientes.

\section{b. Mercados}

- Establecer el mercado total para cada producto/servicio, en dinero y unidades.

- Establecer índices de crecimiento actuales de estos mercados.

- Identificar la participación en cada uno de estos mercados.

- Identificar los mercados, territorios o segmentos que no esta alcanzando la empresa. Exponer las razones para ello.

\section{c. Fuerzas macro ambientales}

Consiste en identificar las tendencias de situaciones o factores actuales que están ocurriendo en el entorno del mercado que pueden significar para la empresa ya sea oportunidades o amenazas.

Estos factores pueden estar relacionados con:

- Tecnologia

- Variables demográficas (tasas de nacimientos y mortalidad, distribución por edades y zonas geográficas etc.)

- Variables económicas (distribución del ingreso, ingreso dispo. nible, niveles de ahorro, etc.)

- Variables ecológicas (suministro y costo de los recursos natura. les y energéticos, problemas del deterioro medioambiental, etc. 
- Variables políticas (regulaciones gubernamentales, decretos, leyes que podrian afectar a la empresa y/o industria, etc.)

- Variables culturales (cambios en los valores culturales y estilos de vida de los consumidores actuales y potenciales).

- Variables de suministro (proveedores, materias primas, poder de negociación, etc.)

Dependiendo de la complejidad del entorno cada empresa tiene que identificar cuáles son las tendencias que le afectan o podrian afectarle.

\section{d. Productos}

- Puntos fuertes y débiles de nuestros productos/servicios.

- Evaluación objetiva, sobre aspectos tales como:

- Calidad

- Rendimiento

- Ventaja diferenciales

- Precio

- Presentación

- Satisfacción del consumidor, etc.

- Ciclo de vida de productos (análisis de cartera)

- Actividades de merchandising

e. Competencia

- Mapa de todos los competidores con influencia significativa en el mercado.

- Sectores que están trabajando.

- Fortalezas y debilidades

- Todo tipo de información de trabajo pertinente al desarrollo de la competencia (ventas, precios, publicidad, etc.)

\section{f. Distribución - Fuerza de ventas}

- Canales de distribución de nuestros productos y su capacidad 
de penetración.

- Imagen de presencia.

- Cobertura de mercados.

- Análisis de la fuerza de ventas (territorios, zonas, capacidades, número, etc.)

- Análisis de las ventas, por caracteristicas de interés.

\section{g. Actividades del consumidor}

- Análisis de actitudes del consumidor hacia la organización y su gama de productos.

- Determinar qué caracteristicas son más importantes para los consumidores.

- Evaluación de la imagen vendida; una imagen entregada.

- ¿Están los productos/servicios satisfaciendo las necesidades?

\section{h. Comunicación al consumidor}

Consiste en hacer una evaluación de las actividades de la mezcla comunicacional de la empresa (promoción de ventas, relaciones públicas, publicidad y venta personal), para ver si estamos maximizando los siguientes aspectos:

- Fijación de los prospectos

- Medios de comunicación

- Confiabilidad de los desembolsos en medios

- La conciencia de los prospectos de que existimos y de lo que ofrecemos.

- La sinergia (hacer que la estrategia de comunicación, desempeñe dos o más funciones al mismo tiempo).

- La vinculación (motivación real de los prospectos a la compra).

- En sentido global todas las actividades comunicacionales ¿Están orientadas a nuestros públicos objetivos?

Para muchos de los aspectos relacionados con la información del análisis de situación se deberá recurrir a las técnicas de inves- 
tigación de mercados tales como:

- Prueba de productos

- muestreo de productos

- investigación de nuevos productos

- selección de conceptos de nuevos productos

- investigación de la publicidad

- prueba de empaques y nombres de marca

- investigación de imagen e identidad

- chequeos de distribución

- investigación de medios y monitoreo, etc.

\section{c. Análisis de producto - mercados}

El análisis situacional permite a la empresa y a los que toman decisiones en especial en el área de mercadeo, identificar oportunidades y amenazas. Primordialmente nos ayuda a definir o a redefinir si fuera necesario el área producto-mercado a través del análisis de tendencias obtenido de los datos básicos.

Un área producto-mercado se define como una combinación de personas con necesidades que los conducen a solicitar algo, por un lado, y por el otro, ciertos beneficios a través de productos que satisfacen esas necesidades entonces un producto-mercado es una combinación de beneficios, de productos con necesidades de mercado que conducen a su solicitud.

Las actividades claves relacionadas con los productos-mercados incluyen:

- Definición de producto-mercados

- Investigar acerca de las necesidades y características de las personas u organizaciones dentro de cada producto-mercado.

- Estimación de la magnitud del Producto-mercado y pronósticos de la forma en que cambiará en el futuro.

- Análisis de la industria y de la estructura de la competencia en el producto mercado, para identificar oportunidades y evitar riesgos. 
En esta etapa, lo que se pretende es definir o redefinir en forma correcta los mercados, con el objeto de analizarlos y pronosticar tendencias futuras. No es muy útil en la planificación mercadológica hacer referencia a un mercado en términos demasiado amplios.

En el momento de definir el área producto-mercado se pueden distinguir los siguientes niveles:

- Producto-mercado genérico, que incluye todos los productos o servicios que pueden satisfacer una necesidad específica. Ej. productos para satisfacer las necesidades que las personas tienen que comer fuera de casa.

- Producto-mercado especifico que representa a todas las marcas de una categoría específica de productos. Ej. Restaurantes, hoteles, cafeterías.

- Producto-mercado de marcas, que son las marcas que compiten entre si. Ej. Mc Donald's, Hotel Camino Real, Doña Mercedes, etc.

\section{Quía básica para el análisis de mercados}

- ¿Se han definido los productos-mercado que se están atendiendo?

- ¿Quiénes son nuestros clientes existentes y potenciales?

- ¿Cuáles son las caracteristicas de nuestros clientes?

- ¿Qué factores influyen sobre las decisiones de compra?

- ¿Qué tan grande es nuestro mercado y con qué velocidad está creciendo?

- ¿La información anterior sugiere algún cambio en nuestros programas mercadotécnicos?

\section{d. Determinar mercado objetivo}

Una vez contestadas las preguntas de las etapas anteriores generalmente se llega a la conclusión que no todos los compradiores son iguales. Diferentes grupos de compradores probablemente respondan de diferentes maneras a los precios, a las promociones, a sus caracteristicas, etc. 
La decisión del mercado objetivo es la elección de a qué personas u organizaciones dirigirá la empresa su programa de mercadotecnia.

Las interrogantes típicas con las que se encuentran las empresas en esta área, es decidir si atender a todos los clientes que estén dispuestos a comprar o debe dirigirse en forma selectiva a uno o varios grupos de clientes. Para tomar una decisión con respecto al mercado objetivo es necesario comprender el producto-mercado.

Hay dos opciones básicas a tomar en cuenta para definir el mercado objetivo o mercado target.

- Estrategia masiva. Se asume que todos los clientes potenciales de un producto-mercado, son suficientemente similares en cuanto a su respuesta a un programa de mercadeo.

- Estrategia de nicho. Se asume que las personas u organizaciones dentro de un producto-mercado variarán con respecto a su respuesta a cualquier programa de mercadeo.

En esta etapa se identifica una posición deseada en un mercado determinado (tomando como base las etapas anteriores). Aqui establecemos:

- ¿Qué queremos alcanzar?

- ¿Hacia quién deberá ir dirigido nuestro esfuerzo mercadotécnico?

Por ejemplo, si estamos operando en un mercado de compradores industriales, hay que definir:

- Tipo de industria

- Actividades específicas

- Productos elaborados

- ¿Qué están comprando?

- ¿Para qué lo están comprando?

- Etc.

Si estamos operando en un mercado de consumo final deberemos identificar: 
- Datos económicos

- Datos demográficos (edad, sexo, etc.)

- Datos geográficos

- Datos sicográficos

- Necesidades, deseos y actitudes, etc.

e. Desarrollo de estrategias (producto, precio, plaza y promoción)

La elección de una estrategia de mercado objetivo es sólo uno de los aspectos del plan de mercadeo, la otra mitad consiste en elegir un programa de mercadotecnia apropiado para satisfacer las necesidades de los clientes y para lograr los objetivos de mercadeo de la empresa. En este sentido se tiene que contar con objetivos bien definidos de manera que sean relevantes y consistentes.

\section{e.1 Estrategia de producto}

La estrategia de producto es la parte central de la planificación de una empresa y juega un papel primordial en la elaboración de la estrategia de mercadotecnia, dado que la función esencial de todo producto o servicio es la de satisfacer una necesidad o deseo de consumidor.

La estrategia de producto consiste básicamente en:

1. Definir los atributos del producto, que son las principales caracteristicas que exigen los compradores o usuarios del producto.

2. Determinar cómo queremos posicionar el producto.

3. Definición del producto, ¿qué características debe tener nuestro producto para ocupar un lugar óptimo?

4. Decidir la forma de colocar una oferta de producto de una unidad de negocio. (Un producto específico, línea o mezcla) para atender su mercado objetivo.

5. Fijar objetivos estratégicos para la oferta del producto.

6. Elegir una estrategia de marca.

7. Elaborar e implantar una estrategia de administración para productos nuevos y existentes. 
Una de las decisiones más importantes que se debe tomar sobre los productos es el establecimiento de prioridades para cada uno de ellos con el objetivo de orientar la planificación de los productos.

Existen tres importantes decisiones de planificación de productos que deben tomarse:

1. ¿Se debe elaborar un nuevo producto?

2 ¿Se debe mejorar el producto?

3. ¿Se debe eliminar un producto?

Estas decisiones y las actividades necesarias para llevarlas a cabo forman la parte crucial de la planificación de productos en una empresa.

Algunas posibles estrategias de producto

- Agregar algo, cambiarlo, modificarlo, mejorarlo.

- Ampliar o consolidar la línea.

- Agregar accesorios.

- Ofrecer nuevas gamas de colores.

- Hacerlo más seguro.

- Aumentar su calidad.

- Incrementar su vida o eficiencia.

- Introducir nuevos productos.

- Relanzar un producto.

- Rediseñarlo para ahorrar tiempo o dinero al consumidor.

- Hacerlo más compatible con la gama de accesorios ofrecida por la competencia.

- Comprar derechos para fabricar bajo otras marcas, mediante licencia.

\section{e.2 Estrategia de precios}

El precio no es una función aislada, cuya fijación se define únicamente pensada en la rentabilidad. El margen de rentabilidad deseado es un factor importante, preponderante, pero no el único. 
La estrategia de fijación de precios incluye:

1. Determinación de objetivos de fijación de precios, puesto que el precio puede estar directamente relacionado con cada tipo de precios, debe reflejar claramente la estrategia de mercadeo que ha sido seleccionada; en ese sentido se pueden perseguir algunos de los siguientes objetivos:

- Incrementar la tasa de compra para una forma de producto.

- Incrementar la demanda entre no usuarios.

- Retener a los clientes actuales.

- Retener a los clientes rentables.

- Captar nuevos clientes interesados en los precios.

- Captar nuevos clientes interesados en la calidad.

2. Estimación de la elasticidad.

La capacidad de lograr el objetivo del precio dependerá de la elasticidad de la demanda. La comprensión de los factores que determinan la elasticidad puede proporcionar linamientos generales para calcularla adecuadamente.

3. Estimación de los costos de producción y comercialización del producto.

Los costos fijos y variables son los intereses más grandes del fijador de precios. Es más, el fijador de precios puede a veces considerar otros tipos de costos, como costos de oportunidad, costos incrementales y costos de reemplazamiento.

4. Seleccionar un programa de fijación de precios.

En la práctica estos programas se basan generalmente en una orientación al mercado o en una orientación a la utilidad.

- Orientación hacia el mercado: se consideran los costos y la rentabilidad, pero la base fundamental para la selección del programa es la elasticidad de la demanda.

- Orientación hacia la rentabilidad: son aquellos en los que se tienen en cuenta fundamentalmente los costos y los margenes 


\section{de utilidad.}

5. Estrategia de fijación de precios para productos nuevos.

Encontramos dos tipos básicos de estrategias: precios de desnatación (establecer un alto precio inicial para un producto con una visión de desnatar la crema del mercado en el extremo superior de la curva de demanda) y precios de penetración (entrar al mercado con un precio inicial bajo para poder captar una porción más grande del mercado.)

6. Estrategia de fijación de precios para productos establecidos.

Aqui tenemos las siguientes alternativas: mantener el precio, reducir el precio e incrementar el precio.

\section{Estrategias de flexibilidad.}

Esta estrategia consiste en dos alternativas: la política de un precio único y la estrategia de precio flexible. La primera significa que el mismo precio es fijado para todos los clientes que compran artículos en las mismas condiciones y en las mismas cantidades. La segunda se refiere a situaciones en las que los mismos productos y/o cantidades son ofrecidas a diferentes clientes a precios diferentes.

\section{e.3 Estrategia de plaza (Distribución)}

Plaza es colocar el producto de la manera más eficiente posible al alcance del consumidor o usuario incluyendo los servicios postventa. El propósito a este respecto es que se logre hacer más fácil para el consumidor o usuario los siguientes aspectos:

- Adquirir el producto

- Obtener información o asistencia técnica

- Solucionar problemas con su uso

- Usarlo

- Operarlo

- Darle mantenimiento, repararlo, etc. 
Existen dos decisiones básicas sobre distribución:

1. Decidir cómo llegar a los mercados objetivos.

2. Si se decide utilizar intermediarios en vez de participar en forma directa, elegir el papel que la empresa jugará en el canal y la red de canales de la que se convertirá en parte.

Los factores que afectan a la estrategia de distribución son: consideraciones del usuario final, características del producto y consideraciones financieras y de control.

\section{Estrategias básicas de distribución}

Existen tres tipos de estrategias básicas de distribución:

\section{Estrategia selectiva.}

Esta se basa en elegir en una zona geográfica-comercial a una minoría idónea para la distribución de un producto. No se pretende alcanzar una distribución total.

\section{Estrategia exclusiva.}

Es un tipo de distribución selectiva, sólo que en cada área geográfica-comercial solamente un establecimiento o empresa comercial, o a la suma dos pueden vender el producto.

\section{Estrategia intensiva.}

Esta estrategia tiene por objeto situar el producto en todos los puntos de venta posibles. Básicamente se utilizan en productos de consumo masivo.

\section{Algunas consideraciones estratégicas}

- Cambiar canales de distribución adecuándolo a patrones de actitudes del consumidor.

- Agregar o reducir mayoristas

- Organizar canal de ventas externo o consolidar la fuerza de ventas propia. 
- Establecer sucursales para facilitar la entrega.

- Vender a través de catálogos.

- Hacer que sea más fácil para sus intermediarios el almacenar, manejar o inventariar sus productos.

- Establecer un red de centros de servicios.

- Agregar vendedores, etc.

\section{e.4 Estrategia de comunicación}

\section{Estrategia de relaciones públicas}

T a función de relaciones públicas es el conjunto de actividades Lefectuadas por cualquier organización para la creación y mantenimiento de buenas relaciones entre los miembros de la organización, y entre la organización y los demás sectores de la opinión pública tales como proveedores, clientes, inversionistas, gobierno, público en general, a fin de proyectar entre ellos una imagen favorable de la organización que contribuya al alcance de los objetivos de ésta.

La estrategia de relaciones públicas incluye:

1. Definición y clasificación de los públicos importantes que conforman la opinión.

2. Situación de las relaciones con los diferentes públicos.

3. Definición de objetivos generales de la función.

4. Definición de politicas generales de la función

5. Definición de objetivos específicos para cada público.

6. Definición de las políticas de relación para cada público especifico.

7. Planificación de actividades a realizar y definición de medios de comunicación a utilizar.

Estrategia publicitaria

La publicidad puede ser dividida en dos tipos: 
1. Publicidad Promocional Directa, y

2. Publicidad Institucional.

La publicidad promocional o simplemente publicidad, es la función consistente en dar a conocer en forma masiva al público en general (clientes actuales y potenciales), las características de los productos $y / o$ servicios, a través de medios masivos de comunicación para desarrollar la conveniencia de su consumo o uso, con el fin especifico de incrementar ventas. La publicidad institucional es la que se emplea para tratar de evocar una imagen favorable de una organización y no para vender un producto o servicio determinado. Este tipo de publicidad es uno de los medios de comunicación con que cuentan las relaciones públicas.

La estrategia publicitaria se conforma con los siguientes pasos:

1. Definición del producto/servicio.

2. Definición del mercado objetivo.

- Geográfico

- Demográfico

- Hábitos de medios

- Hábitos de uso/compra

3. Posicionamiento/Identificación (Promesa única de venta)

4. Puntos adicionales de venta.

5. Determinación de la técnica.

6. Determinación de medios globales y específicos.

7. Objetivos Publicitarios.

Clasificación de la publicidad

- Por su contenido

- Promocional

- Institucional

- Por su tipo de demanda

- Demanda Primaria 
— Demanda Selectiva

- Por el público al que se dirige.

- Para el consumidor

- Para el distribuidor

- Por su patrocinador.

- Del fabricante

- Del intermediario

- Cooperativa

\section{Estrategia de ventas}

T a efectividad de una fuerza de ventas es decisiva para el éxito de la mayoría de las estrategias de mercadeo.

Para desarrollar una estrategia de ventas se requiere de cuatro etapas importantes:

1. Definición de objetivos de ventas diseñados para ejecutar la estrategia de mercadeo. Estos pueden ser:

- Objetivos de desarrollo de clientes.

- Objetivos de respaldo del distribuidor.

- Objetivos de mantenimiento de clientes.

- Objetivos de penetración de clientes.

2. Identificar los atractivos de venta más apropiados para alcanzar los objetivos. Los atractivos expresan los beneficios que el vendedor ofrece para obtener el tipo de respuesta que se ha establecido como objetivo del programa. Estos pueden ser:

- Atractivos de producto

- Atractivos logísticos

- Ofertas de protección

- Atractivos de simplificación

- Atractivos de precio 
- Atractivos de asistencia financiera.

3. Determinar y asignar recursos humanos y financieros que requiere la estrategia.

4. Evaluar el desempeño de la estrategia para modificarla cuando sea necesario.

\section{f. Paso de los planes a la acción}

El propósito de esta etapa es elaborar análisis financieros de apoyo para la estrategia de mercadotecnia, con el objeto de mostrar el impacto estimado de la estrategia sobre el desempeño financiero y para señalar los requerimientos financieros de mercadeo durante el periodo de planificación.

\section{g. Seguimiento}

La evaluación o seguimiento, es la última etapa del plan de mercadeo. La planificación de mercadeo requiere información de diversas actividades de revisión y evaluación del desempeño.

Secuencia del proceso de evaluación

- ¿Qué deseamos alcanzar realmente?

- ¿Qué está ocurriendo realmente?

- ¿Por qué está ocurriendo?

- ¿Qué debo hacer acerca de lo que está ocurriendo? 


\section{Bibliografía}

Cravens David

Stapleton J.

Hehman Raymond

Gulltinan Joseph

Subhash Jain

Rapp Stan, Collins Tom

Lambin

Mercado Salvador

Martinez Ezequiel
Planeación en Mercadolecnia. CECSA, 1895.

El Plan de Mdrkeling. Piedra Santa 1990.

Cómo Preparar un Plan de Mdrketing. Deusto

Desarrollo y Ejecución de Estrategias de Mercadeo. NORMA 1986.

Administración de Mercadeo. Mc. Graw Hill, 1988.

Dirección de Mórketing. Casa Nueva.

Maxi Márketing. Mc. Graw Hill, 1988.

Mdrketing Estratégico. Mc. Graw Hill, 1987.

Mercadotecnia Programada. LIMUSA, 1987.

Planeación, Desarrollo e Ingenieria de Producto. TRILLAS, 1987.

\section{Revistas}

La Planificación como Aprendizaje. HarvardDeusto, Business Review N' 36, Cuarto trimestre, 1988.

Arie P. de Geas

La Promoción de Venlas en el Mörketing de Hoy. Harvard-Deusto, Business Review ND 35, Tercer Trimestre, 1988.

Arie P. de Geas

Sisteme de Distribucion Basados en el Consumidor. Harvard-Deusto, Business Review No 34, Segundo Trimestre, 1988.

Antonio Menéndez

El Comportamiento del Consumidor. Admón. y Empresa N 40, abril-junio, 1988.

Interacción de las Politicas de Selección de Canales de Distribución en el Sistema de Märketing. Admón. y Empresa NN 35, Enero-marzo, 1988.

Majluf Nicolas
El Uso de la Matriz - Participación en la Planención. Admón. y Empresa No 32, Abriljunio, 1987. 


\section{Camará Dionisio}

Montafía Jordi

Santos Jorge

Montafha Jordi

Barraza Picone, Sergio
El Desarrollo del Concepto Márketing y sus Herramientas de Gestión. Admón. y Empresa N ${ }^{\mathrm{P}}$ 33, Julio-Septlembre, 1987.

Diseño y Estrategia de Producto. Admón. y Empresa № 33, Julio-Septiembre, 1987.

: $\quad$ Relevancia del Plan de Mórketing. Para la Pequeña y Mediana Empresa. Admón. y Empresa N 25, Octubre-Diciembre, 1985.

Politice de Marketing: El Producto. Admón. y Empresa N2 26, Octubre-diciembre, 1985.

Necesidades y Tipos de Información de las Actioidades de Márketing. Admón. y Empresa N²6, Julio-Septiembre, 1985. 